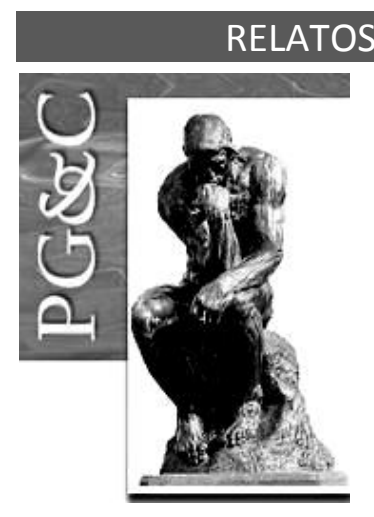

\title{
A PERSPECTIVA DA RELAÇÃO ENTRE ESTILOS DE APRENDIZAGEM E A ESTRUTURA DE MAPA CONCEITUAL
}

\author{
Franciene Duarte Gomes \\ Mestra em Ciência da Computação pelo Centro Universitário Eurípides de \\ Marília, Brasil. Professora da Universidade Federal do Mato Grosso do Sul, \\ Brasil. \\ E-mail: franciene@gmail.com
}

Juan Fernando Galindo Jaramillo

Mestre em Tecnologia pela Universidade Estadual de Campinas, Brasil. Professor da Fundação Hermínio Ometto, Brasil.

E-mail: jgalindoj@gmail.com

\section{Wagner José da Silva}

Especialista em Informática em Educação pela Universidade Federal de Lavras, Brasil. Professor do Centro Estadual de Educação Tecnológica Paula Souza, Brasil.

E-mail: wjsilva2302@gmail.com

\section{Gisele Busichia Baioco}

Doutora em Ciência da Computação e Matemática Computacional pela Universidade de São Paulo, Brasil. Professora da Universidade Estadual de Campinas, Brasil.

E-mail: gisele@ft.unicamp.br

\section{Antonio Carlos Zambon}

Doutor em Engenharia de Produção pela Universidade Federal de São Carlos, Brasil. Professor da Universidade Estadual de Campinas, Brasil. E-mail: zambon@ft.unicamp.br

\section{Resumo}

Nos ambientes de ensino, existem alunos com diferentes estilos de aprendizagem dominantes. Identificar esses estilos em uma turma facilita ao professor ajustar estratégias de ensino-aprendizagem considerando as preferências dos alunos. Geralmente a identificação dos estilos de aprendizagem baseia-se em questionários longos, causando desmotivação nos alunos por causa do tempo de preenchimento. Este trabalho propõe o uso de mapas conceituais para a identificação de estilos de aprendizagem. Os mapas conceituais permitem a representação do conhecimento de um indivíduo em um domínio. A identificação do estilo de aprendizagem dominante a partir de mapas conceituais facilitaria ajustar estratégias de ensino-aprendizagem a esses estilos. Assim, o objetivo deste estudo foi identificar uma conexão entre o estilo de aprendizagem dominante e a métrica de complexidade de mapas conceituais, criados a partir de textos dos alunos. Para isso, foi feito um estudo em uma turma de graduação que respondeu uma questão dissertativa. A partir da resposta de cada aluno foi criado um mapa conceitual usando uma técnica chamada Verbka. Também foi identificado o estilo de aprendizagem dominante de cada aluno usando o questionário VARK. Com esses dados, foi comparada a complexidade dos mapas conceituais com o estilo de aprendizagem dominante de cada aluno. $\mathrm{O}$ principal resultado do estudo apontou relação entre estilo de aprendizagem e a métrica de complexidade dos mapas dos alunos desse estilo. Assim, concluímos que é possível identificar a

Perspectivas em Gestão \& Conhecimento, João Pessoa, v. 8, número especial, p. 118-134, out. 2018. DOI: http://dx.doi.org/10.21714/2236-417X2018v8nep118

http://periodicos.ufpb.br/ojs2/index.php/pgc. ISSN: 2236-417X. Publicação sob Licença (cc) EY-NC-ND 
proporção de estilos de aprendizagem dominantes da turma com base em textos dos alunos, facilitando o monitoramento dos estilos de aprendizagem, sem atividades adicionais.

Palavras-chave: Mapas de Conhecimento; Métrica de Complexidade do Mapa; Teoria VARK.

\title{
A PERSPECTIVE OF THE RELATIONSHIP BETWEEN LEARNING STYLES AND CONCEPT MAP STRUCTURE
}

\begin{abstract}
There are differences among students in teaching environments, related to their dominant learning style. The identification of those styles in a classroom eases the adjusting of learning-teaching strategies considering students' preferences. Learning styles identification is usually based on long questionnaires, causing demotivation in students due to the time spent in questionnaire filling. This work proposes the use of concept maps for the identification of learning styles. Concept maps allow individual's knowledge representation on a given domain. The identification of the dominant learning styles from concept maps could ease the adjustment of teaching-learning strategies to those styles. Thus, the objective of this study was the identification of a connection between students' dominant learning style and the complexity metric of concept maps created from texts made by the students. For that purpose, a case study was made with a bachelor course class whose students answered a dissertational question. From each student's answer to that question, a concept map was created using a technique called Verbka. Students' dominant learning style was also identified using the VARK questionnaire. With those data, concept maps' complexity was compared with students' dominant learning style. The main result of this study indicates a relationship between the dominant learning style and the complexity metric of the concept maps for students with that dominant style. Therefore, we conclude that it is possible to identify the proportion of dominant learning styles present in a classroom from students' texts, easing the monitoring of learning styles without performing additional activities.
\end{abstract}

Keywords: Knowledge Maps. Map Complexity Metric. VARK Theory.

\section{INTRODUÇÃO}

O processo de construção de conhecimento está passando por uma era de mudança nos modos de ensinar e aprender. Respondendo a essa característica, a Engenharia do Conhecimento foca no desenvolvimento de modelos e técnicas computacionais voltadas para a modelagem, representação e processamento do conhecimento (REZENDE, 2003). Dentre as técnicas disponíveis estão os mapas de conhecimento, muito utilizados por serem uma maneira eficiente de estruturar, organizar e representar o conhecimento (EPPLER, 2008).

Existem diversos tipos de estruturas de mapas de conhecimento (BALAID et al., 2016). Dentre esses tipos, têm-se os mapas conceituais (NOVAK; CAÑAS, 2008), cuja estrutura é formada por conceitos e seus relacionamentos que formam proposições. Com essa estrutura, representa-se o conhecimento de uma pessoa ou de uma organização em um determinado domínio. Segundo Balaid et al. (2016), o mapa conceitual é a melhor técnica para visualizar as relações entre os conceitos e explicitar o conhecimento de uma pessoa sobre um domínio. Portanto, são utilizados em ambientes de aprendizagem como estratégias de ensinoaprendizagem (AGUIAR; ROGÉRIO; CORREIA, 2013; CICUTO; MENDES; CORREIA, 2013; HASSANZADEH et al., 2016; KICKEN et al., 2016). Todavia, existem diferenças entre os alunos em decorrência de diversos fatores que podem afetar o resultado das estratégias de ensinoaprendizagem, e entre esses estão os estilos de aprendizagem e os estilos cognitivos.

Considerando as várias definições de estilos de aprendizagem, Keefe (1979) destaca as características cognitivas, afetivas e psicológicas do indivíduo como necessárias para a aprendizagem. Já os estilos cognitivos representam as preferências individuais, o modo pelo

Perspectivas em Gestão \& Conhecimento, João Pessoa, v. 8, número especial, p. 118-134, out. 2018. 
qual uma pessoa descobre, processa e avalia os dados, que interfere na maneira como ela organiza, integra e interpreta a informação (ALLINSON; HAYES, 1996).

Mesmo relacionados, a diferença entre os estilos cognitivos e os estilos de aprendizagem está em que os primeiros representam a organização de processos cognitivos, que levam uma pessoa a tarefas de aprendizagem como pensar, aprender e resolver um problema. Já os estilos de aprendizagem se relacionam com as preferências de aprendizagem, ou seja, a maneira como um conteúdo pode ser melhor aprendido. Alguns autores consideram os estilos de aprendizagem como um subconjunto dos estilos cognitivos, com aspectos afetivos e psicológicos (HAYES; ALLINSON, 1994; RIDING; S.RAYNER, 2013). Este trabalho foca nos estilos de aprendizagem, mantendo em consideração sua relação com os estilos cognitivos.

Existem diferentes modelos de estilos de aprendizagem, dependendo da teoria que suporta o modelo e o tipo de preferência a ser identificado. Neste trabalho, será utilizado o modelo VARK. Para Fleming (2001), os estilos de aprendizagem, conforme a teoria VARK (Visual, Aural, Read/Write e Kinesthetic), se focam nos canais sensoriais/percepção, em relação à maneira como as pessoas processam a informação.

Usualmente, os estilos de aprendizagem são identificados por meio de questionários (OCEPEK et al., 2013). Esses questionários contam com alta confiabilidade, pelo fato dos resultados fornecerem um valor ajustado para cada estilo, indicando o modo de aprendizado do respondente. Porém, alguns questionários são muito longos, o que torna seu preenchimento demorado (FELDMAN; MONTESERIN; AMANDI, 2015; OCEPEK et al., 2013), podendo causar desmotivação nos alunos (BERNARD et al., 2015).

A demora no preenchimento dos questionários cria outro desafio na sala de aula, já que há uma variação dos estilos de aprendizagem com o passar do tempo (KIRSCHNER, 2017). Considerando essa característica, para poder ajustar o processo de aprendizagem às mudanças nos estilos de aprendizagem, deve-se encontrar uma maneira de identificar esses estilos continuamente. Essa identificação deve ser executada da maneira menos invasiva possível, evitando ao máximo atividades adicionais para alunos e professores.

Nesse aspecto, ferramentas de Engenharia do Conhecimento podem ser úteis em um ambiente de ensino, a fim de apoiar na identificação dos estilos de aprendizagem presentes em uma turma. Dentre essas ferramentas, o processo Verbka (VASQUES et al., 2016), baseado na extração de conhecimento contido em textos, permite a extração de proposições e conceitos de uma questão discursiva, convertendo-os em um mapa conceitual. Como os mapas conceituais resultantes são formados por conceitos e proposições formadas por esses conceitos presentes no texto, esses mapas podem revelar a estrutura conceitual do autor. Se a estrutura conceitual estiver relacionada com o estilo de aprendizagem dominante, então seria possível a criação de uma técnica para a identificação do estilo de aprendizagem do aluno através de textos elaborados em atividades da disciplina.

Partindo do pressuposto anterior, o objetivo deste estudo foi identificar se há alguma relação entre o estilo de aprendizagem e a complexidade de um mapa conceitual criado a partir de textos escritos por alunos de uma disciplina. Assim, poderiam ser consideradas as mudanças nos estilos de aprendizagem usando atividades que os alunos fazem na sala de aula, evitando atividades adicionais. Portanto, propomos como hipótese que a estrutura conceitual do mapa conceitual de um aluno tem relação com o seu estilo de aprendizagem. Para a identificação dessa relação, aplicamos a métrica de complexidade de mapa conceitual (DEFRANCO et al., 2012), representada pelo número de proposições e número de conceitos dos mapas obtidos usando o processo Verbka.

Este artigo está organizado em seis seções, além desta introdução: na Seção 2 apresentam-se os trabalhos relacionados; na Seção 3, a base teórica utilizada para a construção do trabalho; na Seção 4 descrevemos a metodologia; a Seção 5 apresenta os resultados; a discussão dos resultados está presente na Seção 6; por fim, a Seção 7 contém as

Perspectivas em Gestão \& Conhecimento, João Pessoa, v. 8, número especial, p. 118-134, out. 2018. 
conclusões e a proposta de trabalhos futuros.

\section{TRABALHOS RELACIONADOS}

No desenvolvimento da pesquisa, procuramos artigos que descrevessem a relação entre estilos de aprendizagem e técnicas de mapeamento conceitual, encontrando uma lacuna na literatura. Por esse motivo, a pesquisa bibliográfica foi ampliada para trabalhos que relacionassem estilos cognitivos e mapeamento conceitual. Essa pesquisa bibliográfica revelou os trabalhos de Graff (2005), DeFranco et al. (2012) e Stoyanov et al. (2017) como relevantes.

Graff (2005) estudou a relação do uso de mapas conceituais em hipertexto com a teoria de estilos cognitivos de Allinson e Hayes (1996). O estudo foi aplicado em uma turma com 55 participantes que tiveram que lembrar uma dada informação e elaborar mapas de hipertexto com base nessa informação. $O$ autor desse estudo aplicou a métrica de densidade representada pela fórmula ligações/nós -1 proposta por Klein e Cooper (1982) e a métrica de complexidade proposta por Johnson et al. (1986) representada pela fórmula ligações/nós, sendo que em ambos os casos cada ligação é uma proposição. Como resultado, Graff (2005) aponta que os mapas conceituais elaborados pelos diferentes estilos cognitivos possuem arquitetura de mapa diferente (linear, hierárquico, rede). Ainda segundo o autor, esses resultados podem ter influência na facilidade que alguns participantes poderiam ter na utilização de hipertexto.

DeFranco et al. (2012) estudaram as correlações entre os estilos cognitivos e um conjunto de métricas em alunos de engenharia. As métricas são classificadas como tradicionais (que identificam o número de elementos do mapa) e holísticas (que tratam qualitativamente a compreensão do mapa conceitual). Para isso, foram aplicadas técnicas de mapeamento conceitual "aberto", em que o construtor do mapa pode utilizar seus próprios conceitos, e "fechado", em que o construtor do mapa fica restrito a uma lista de conceitos. Para a identificação do estilo cognitivo foi utilizada a teoria KAI. Essa teoria, proposta por Kirton (1976) foca na criatividade das pessoas, ou seja, em estilos relacionados ao modo de pensar e trabalhar de maneira criativa. KAl significa Kirton Adaption-Innovation Inventory (Inventário de Adaptação-Inovação Kirton). O estudo de DeFranco et al. (2012) foi conduzido com alunos de graduação e pós-graduação de engenharia, aplicando como domínio assuntos não técnicos. Como principal resultado, os autores desse estudo encontraram correlações entre os estilos cognitivos e as métricas aplicadas no modo como os alunos estruturam suas ideias.

Stoyanov et al. (2017) investigaram os resultados do estilo cognitivo para a tomada de decisão em um processo de mapeamento de conceitos de grupo. Os autores também utilizaram a teoria de estilo cognitivo de KAI (KIRTON, 1976). Da mesma maneira, esses autores utilizaram mapas conceituais para representar a construção cognitiva dos participantes do estudo e estabelecer as diferenças entre as pessoas. Como principal resultado, Stoyanov et al. (2017) identificaram que os participantes com estilo adaptativo conseguiram elaborar ideias para um contexto particular. Já aqueles com estilo inovador produziram ideias mais gerais. Outra questão foi a comparação dos mapas utilizando a análise Procrustes (KRZANOWSKI, 1996) que apontou uma dissimilaridade entre os estilos cognitivos.

Os trabalhos encontrados focaram na identificação dos estilos cognitivos com base em técnicas de mapeamento conceitual. Os mapas conceituais foram feitos pelos alunos partindo de uma questão focal. Para a análise dos trabalhos, foram usadas métricas tradicionais e holísticas. No entanto, não foram encontrados trabalhos de mapeamento conceitual focados nos estilos de aprendizagem. Também não foram encontrados trabalhos utilizando uma técnica em que os mapas conceituais fossem criados a partir de textos.

Em vista do encontrado na literatura, este trabalho utiliza um processo de aquisição do conhecimento baseado em semântica verbal denominado Verbka, proposto por Vasques et al.

Perspectivas em Gestão \& Conhecimento, João Pessoa, v. 8, número especial, p. 118-134, out. 2018. 
(2016). Esse processo extrai proposições e revela características do conhecimento baseadas em ações e eventos. Para a representação do conhecimento, Verbka utiliza um mapa conceitual causal estruturado por conceitos, que mostra a relação de causa e efeito em uma proposição. Verbka foi usado para criar mapas a partir de textos escritos por alunos. Os mapas resultantes foram analisados para determinar se há alguma relação entre o estilo de aprendizagem e a complexidade do mapa.

\section{BASE TEÓRICA}

Nesta seção, descrevemos brevemente os conceitos de aquisição do conhecimento, enfatizando os mapas conceituais e o modelo de estilos de aprendizagem VARK, no contexto da pesquisa.

\subsection{Aquisição e representação do conhecimento}

Os procedimentos de aquisição do conhecimento são elaborados considerando uma ou várias fontes de dados, como vídeos, textos, entrevistas ou questionários (MILTON, 2007; MILTON; CLARKE; SHADBOLT, 2006). Quando a aquisição é direcionada a uma pessoa, essa atividade é denominada de elicitação. Por outro lado, quando a aquisição do conhecimento resulta de documentos denomina-se extração (MASTELLA, 2005).

Após a aquisição do conhecimento, é feita a representação do conhecimento (COOKE, 1994). Esta última pode ser definida como a maneira ordenada de estruturar e codificar o conhecimento para um determinado propósito (REZENDE, 2003). Uma dessas maneiras é o mapa conceitual.

Um mapa conceitual é uma representação gráfica formada pela união entre conceitos conectados por palavras ou frases de ligação, gerando uma afirmação com sentido (NOVAK; CAÑAS, 2008). Os conceitos são conjuntos de informações que se inter-relacionam formando um significado (LIPMAN, 1995). Os conceitos em um mapa são apresentados em retângulos e os relacionamentos entre os retângulos são indicados por uma seta. Esses relacionamentos geram proposições, ou seja, afirmações que podem ser avaliadas como verdadeiras ou falsas. Os mapas conceituais são construídos com base em uma questão focal para determinar o seu assunto central. A resposta para essa questão estará representada no mapa seguindo as ligações entre os conceitos. Todas essas ligações fazem com que o mapa conceitual possa ser entendido como uma rede de proposições.

Dessa maneira, a estrutura do mapa conceitual reflete o modo em que 0 conhecimento do domínio é estruturado pelo autor. A Figura 1 mostra a estrutura de uma proposição presente em um mapa conceitual, a qual é constituída por conceitos que têm uma ligação entre si e o termo que os liga.

Figura 1 - Estrutura de uma proposição

\section{Proposição = Conceito Inicial + Termo de ligação + Conceito Final}

Fonte: Elaborada pelos autores

\subsection{VARK}

O modelo teórico de identificação de estilos de aprendizagem VARK (FLEMING, 2001; FLEMING; MILLS, 1992) se foca nos canais sensoriais (audição, visão e cinestésico) preferidos

Perspectivas em Gestão \& Conhecimento, João Pessoa, v. 8, número especial, p. 118-134, out. 2018. 
pelo indivíduo quando aprende. Nesse modo são identificados quatro estilos de aprendizagem: visual, auditivo, leitor/escritor e cinestésico - Visual, Aural, Read/Write e Kinesthetic (VARK). Segue a explicação de cada um desses estilos:

Visual: esse estilo enquadra as pessoas que compreendem melhor por meio da visão e gostam de simbolismo, fontes e cores para destacar os pontos importantes, imagens, fluxogramas e slides entre outros.

> Auditivo: nesse estilo se enquadram as pessoas que compreendem melhor por meio da audição e gostam de instruções faladas, discussões em sala de aula, ouvir sua própria voz, seminários, provas orais, entre outros.

> Leitor/escritor: estilo que enquadra pessoas que gostam de leituras e compreendem melhor fazendo anotações, resumos e esquemas, entre outros.

$>$ Cinestésico: enquadra pessoas que compreendem melhor criando, fazendo movimento e realizando interações, estudos de casos, aulas e trabalhos práticos, laboratórios, entre outros.

O modelo VARK também foca nas combinações dos estilos de aprendizagem, podendo caracterizar um quinto estilo, o multimodal. $O$ estilo de aprendizagem multimodal enquadra pessoas que apresentam preferências de aprendizado de mais de um estilo simultaneamente (VARK-LEARN, 2018). As mesmas estratégias de ensino-aprendizagem que correspondem aos seus estilos, podem ser combinadas para atender ao estilo de aprendizagem multimodal (VARK-LEARN, 2018). Outro ponto sobre o modelo VARK é que o questionário não possui um número excessivo de questões (máximo 20) e o conteúdo das questões está voltado para situações da vida real, o que facilita o entendimento por parte do respondente (VARK-LEARN, 2018).

Assim, se for possível encontrar uma maneira de identificar estilos de aprendizagem presentes em uma turma conforme o modelo VARK, a partir de documentos criados pelos alunos em suas atividades em sala de aula, seria possível para o professor adequar suas práticas de ensino constantemente. Este trabalho aponta à utilização de uma métrica de complexidade de mapas conceituais (DEFRANCO et al., 2012) criados a partir de textos escritos por alunos.

\section{METODOLOGIA}

Para comparar os estilos de aprendizagem dos alunos com mapas conceituais obtidos a partir de textos desses alunos, foi desenvolvido um estudo seguindo uma abordagem de pesquisa quantitativa com aplicação de um estudo de caso ${ }^{1}$. Esse estudo foi aplicado na disciplina de Teoria Geral de Sistemas em um curso de Bacharelado em Sistemas de Informação. A disciplina faz parte do terceiro semestre do curso e tem como pré-requisito a disciplina de Administração de Empresas. Para participar da pesquisa, os alunos deveriam ter cursado também a disciplina de Economia e Finanças, oferecida no segundo semestre do curso. O estudo contou com trinta e sete participantes. Para a construção dos mapas conceituais, escolhemos o uso do processo Verbka descrito na seção a seguir.

\subsection{Verbka}

Verbka é um processo de aquisição do conhecimento baseado na extração de conhecimento presente em textos, fundamentado em semântica verbal (VASQUES et al., 2016).

\footnotetext{
${ }^{1}$ Projeto de pesquisa aprovado pelo Comitê de Ética em Pesquisa com número: 54042816.8.0000.5404.
} 
Os conceitos extraídos e seus relacionamentos explicam os eventos (ações) contidos na narrativa. Esses eventos são então representados em um mapa conceitual causal, ou seja, um mapa com foco nas relações de causa e efeito existente entre conceitos.

O processo é composto por oito etapas, explicadas a seguir (VASQUES et al., 2016):

1. Seleção e preparação do texto: nessa etapa são selecionados e pré-processados os textos dos quais se deseja extrair conhecimento;

2. Seleção verbal: nessa etapa todos os verbos existentes devem ser selecionados pelo usuário;

3. Descoberta do agente e do paciente: deve-se perguntar a cada um dos verbos "Quem?" e "O quê?". A resposta à primeira pergunta revela o sujeito e, a resposta da segunda pergunta revela o objeto da frase. Essas respostas formarão uma tripla no formato sujeito-verbo-objeto.

4. Classificação dos sintagmas: as triplas extraídas da etapa anterior serão inseridas em uma tabela formada por 2 colunas. A primeira coluna diz respeito ao Sintagma Nominal (SN), formada pelo sujeito e seus adjetivos (quando existirem). A segunda coluna traz o Sintagma Verbal (SV), formado pelo verbo e seus complementos (objetos e adjuntos adverbiais);

5. Definição dos papéis temáticos: para formar blocos conceituais ainda menores, cada um dos diferentes complementos verbais (presentes na segunda coluna da tabela dos sintagmas) deve-se perguntar ao verbo: "O quê?", "A quem?", “Onde?" e "Quando". Cada uma dessas respostas constitui na criação de uma nova coluna a ser adicionada na tabela. Todos os conceitos que estiverem na primeira coluna serão definidos como "agente", todos os outros (exceto o verbo), serão definidos como "pacientes".

6. Agrupamento de conceitos semelhantes: deve-se selecionar em cada uma das colunas (exceto a coluna dos verbos) os conceitos que sejam sinônimos. Dentre os sinônimos, deve-se selecionar o conceito mais significativo (genérico), que substituirá os conceitos semelhantes. Esse conceito mais genérico será denotado como "conceito sigma" (C $\sigma)$;

7. Construção do mapa conceitual: com a tabela finalizada, pode-se transferir todas as proposições criadas para um mapa. Para isso, basta colocar os conceitos em círculos e liga-los por meio de setas direcionadas. Essa etapa representa uma fase de aprimoramento no mapeamento de conceitos, já que também possibilita a inserção de inferências (novas ligações) realizadas pelo usuário.

8. Identificação de relações de causa-efeito: com o uso de cores nas setas é possível diferenciar o tipo de relacionamento existente entre os conceitos. Setas vermelhas indicam mudança de estado do conceito "paciente" causadas pelo conceito "agente". Já as setas azuis indicam que a mudança de estado recai sobre o próprio "agente" da ação. Finalmente, as setas pretas representam relações estáticas (ausência de ação), representadas por verbos de ligação, que descrevem apenas características dos sujeitos e, portanto, não acusam mudança de estado dos conceitos.

O uso do Verbka permite, portanto, a criação de mapas conceituais a partir de textos elaborados pelos alunos no contexto do domínio da disciplina. Havendo uma maneira de evidenciar diferentes estilos de aprendizagem relacionada a mapas conceituais (BARROS et al., 2010), seria possível identificar os estilos predominantes presentes na turma, a qualquer momento, usando as atividades feitas pelos alunos na disciplina. Neste artigo, apresentamos uma possível maneira de identificar esses estilos conforme a teoria VARK.

Perspectivas em Gestão \& Conhecimento, João Pessoa, v. 8, número especial, p. 118-134, out. 2018. 


\subsection{Métodos utilizados para análise}

No estudo, foi considerado exclusivamente o estilo de aprendizagem dos alunos e o texto que eles escreveram. Não foi analisado o nível de conhecimento dos alunos nas análises dos mapas conceituais porque o foco do estudo está na topologia dos mapas. Os mapas conceituais foram criados pelos pesquisadores com base nos textos dos participantes por meio da aplicação do processo Verbka. Para cada mapa conceitual calculamos a razão entre proposições e conceitos (p/c). Essa razão é um indicador da complexidade do mapa (GRAFF, 2005; DEFRANCO et al., 2012; JABLOKOW et al., 2015). A fórmula de complexidade é mostrada na Figura 2.

Figura 2 - Fórmula de Complexidade de Mapa Conceitual

\section{Complexidade do mapa $=$}

Fonte: adaptado de DeFranco et al. (2012)
Total de proposições

\section{Total de conceitos}

Na medida em que a razão $\mathrm{p} / \mathrm{c}$ for maior, serão maiores as conexões entre os diferentes conceitos. A Figura 3 mostra um exemplo de dois mapas com o mesmo número de conceitos. Observa-se que no mapa $A$ há três proposições para quatro conceitos e no mapa $B$ há seis proposições para os mesmos quatro conceitos. $O$ valor da razão $p / c$ para o Mapa $B$ é muito maior do que para o Mapa $A$. Dessa maneira, uma maior razão $p / c$ indica um mapa mais interconectado e, portanto, topologicamente mais complexo.

Figura 3 - Exemplo de cálculo de complexidade para dois mapas conceituais

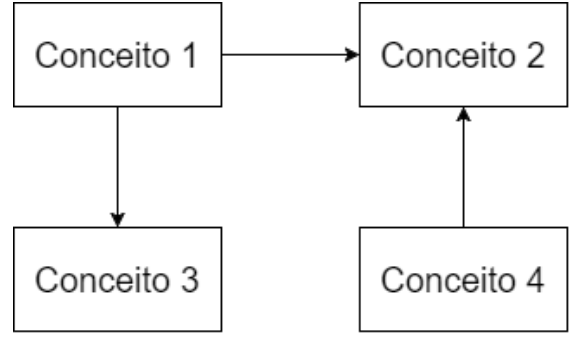

Mapa A:

$\mathrm{p} / \mathrm{c}=0,75$

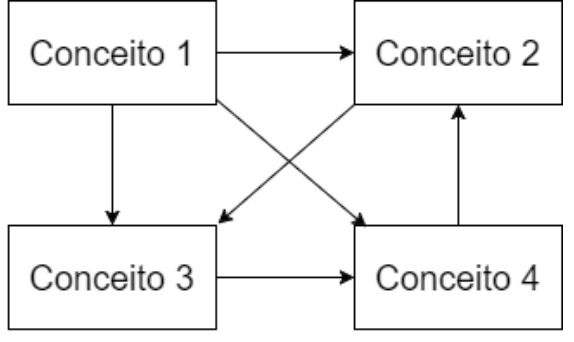

Mapa B:

$\mathrm{p} / \mathrm{c}=1,50$

Fonte: Autores

Em relação às variáveis definidas para o estudo, a variável teórica é a complexidade do mapa conceitual, analisada mediante a razão $\mathrm{p} / \mathrm{c}$, sendo assim a variável dependente. Por sua vez, a variável independente definida foi o estilo de aprendizagem segundo o modelo VARK.

\subsection{Aplicação do estudo}

O professor da disciplina fez uma explanação sobre Economia e Finanças como material de apoio (os alunos da turma tinham cursado anteriormente a disciplina de Economia e Finanças). Desse modo, os alunos responderam a seguinte questão, relacionada com esse domínio:

"Como o cenário econômico afeta os investimentos em empresas de comércio de 
tecidos, vestuários e calçados?".

O formulário da questão tinha no máximo 07 linhas. Essa limitação na resposta foi feita para restringir a quantidade de conceitos e proposições que poderiam ser extraídos pelo Verbka. De acordo com Novak e Cañas (2008), é recomendável em um mapa conceitual manter-se entre 15 e 20 conceitos para facilitar a construção e leitura do mapa. Os alunos também responderam o questionário do modelo teórico VARK (FLEMING; MILLS, 1992). Para a aquisição do conhecimento, aplicamos o processo Verbka para a elaboração dos mapas conceituais seguindo as etapas descritas na subseção 4.1. O Quadro 1 traz o exemplo da transcrição da resposta de um aluno à questão, com estilo de aprendizagem predominante multimodal.

Quadro 1 - Transcrição da resposta de um aluno

Não. No atual cenário econômico brasileiro, com a desaceleração econômica, inflação em alta e desvalorização do real, o comércio em geral não tem obtido resultados satisfatórios. Os noticiários alertando cada vez mais sobre a crise e desemprego são uma parte que influencia o consumidor, que tem priorizado gastar com alimentação e medicamentos, por exemplo, ao passo que quase não tem disposto dinheiro em tecido, vestuário e calçados, razão pela qual esse ramo não está aquecido agora.

Fonte: Autores

O Quadro 2 mostra uma parte da aplicação do processo Verbka, denominada de ProtoPapéis temáticos, no texto do Quadro 1. O Quadro de Proto-Papéis temáticos se divide em dois temas sendo: o Proto-Agente, quem faz a ação, e o Proto-Paciente, quem recebe a ação (VASQUES et al., 2016). Esses procedimentos foram feitos para todos os alunos. A Figura 4 mostra o mapa conceitual resultante do Quadro 2. Os conceitos correspondem às colunas "quem", "o quê/qual", "para quem", "onde" e "quando". As conexões, por sua vez, correspondem aos verbos. Para essa amostra não houveram respostas para a coluna "quando". Assim sendo, essa coluna foi retirada do Quadro 2.

Quadro 2-Quadro de Proto-papéis temáticos

\begin{tabular}{|c|c|c|c|c|c|}
\hline & Proto-Agente & & Proto-I & ente & \\
\hline & & V & Compl. & Compl. & Compl. \\
\hline & "quem" & & "o quê/qual" & $\begin{array}{l}\text { "para } \\
\text { quem" }\end{array}$ & "onde" \\
\hline 1 & $\begin{array}{l}\text { investimentos em empresas } \\
\text { de comércio de vestuário }\end{array}$ & não é & conveniente & & $\begin{array}{c}\text { atual cenário } \\
\text { econômico } \\
\text { brasileiro }\end{array}$ \\
\hline 2 & $\begin{array}{l}\text { atual cenário econômico } \\
\text { brasileiro }\end{array}$ & tem & $\begin{array}{c}\text { desaceleração } \\
\text { econômica }\end{array}$ & & \\
\hline 3 & $\begin{array}{l}\text { atual cenário econômico } \\
\text { brasileiro }\end{array}$ & tem & inflação em alta & & \\
\hline 4 & $\begin{array}{l}\text { atual cenário econômico } \\
\text { brasileiro }\end{array}$ & tem & $\begin{array}{c}\text { desvalorização } \\
\text { do real }\end{array}$ & & \\
\hline 5 & $\begin{array}{l}\text { atual cenário econômico } \\
\text { brasileiro }\end{array}$ & não obtém & $\begin{array}{c}\text { resultados } \\
\text { satisfatórios }\end{array}$ & & \\
\hline 6 & os noticiários & alertam & sobre a crise & & \\
\hline 7 & os noticiários & alertam & $\begin{array}{c}\text { sobre o } \\
\text { desemprego }\end{array}$ & & \\
\hline 8 & os noticiários & influenciam & o consumidor & & \\
\hline 9 & o consumidor & prioriza & $\begin{array}{l}\text { gastos com } \\
\text { alimentação }\end{array}$ & & \\
\hline
\end{tabular}

Perspectivas em Gestão \& Conhecimento, João Pessoa, v. 8, número especial, p. 118-134, out. 2018. 


\begin{tabular}{|c|l|c|c|c|c|}
\hline $\mathbf{1 0}$ & o consumidor & prioriza & $\begin{array}{c}\text { gastos com } \\
\text { medicamento }\end{array}$ & & \\
\hline $\mathbf{1 1}$ & o consumidor & não gasta & dinheiro & & com vestuário \\
\hline $\mathbf{1 2}$ & o consumidor & não aquece & $\begin{array}{r}\text { o ramo do } \\
\text { vestuário }\end{array}$ & & \\
\hline
\end{tabular}

Fonte: Autores

A partir da tabela de Proto-Papéis temáticos (Quadro 2) é construído o mapa conceitual para cada texto. Os conceitos nessa figura estão conectados entre si por verbos mantendo a estrutura das linhas do Quadro 2. Neste trabalho, somente foi utilizado o complemento verbal relacionado à questão "o quê/qual", para manter a estrutura de proposição descrita na subseção 3.1. Observa-se na Figura 4 que o mapa conceitual resultante é composto por dois blocos em lugar de um, como é usual nos mapas conceituais. Essa situação acontece pela existência de relações implícitas (ocultas) entre conceitos, que não foram explicitadas no texto original. Verbka permite a criação de novos conhecimentos, possibilitando ao usuário a inserção dessas conexões. No entanto, neste trabalho não foram exploradas essas inferências já que a métrica de complexidade do mapa seria afetada pela adição de conexões.

Figura 4 - Mapa conceitual resultante de texto usando Verbka

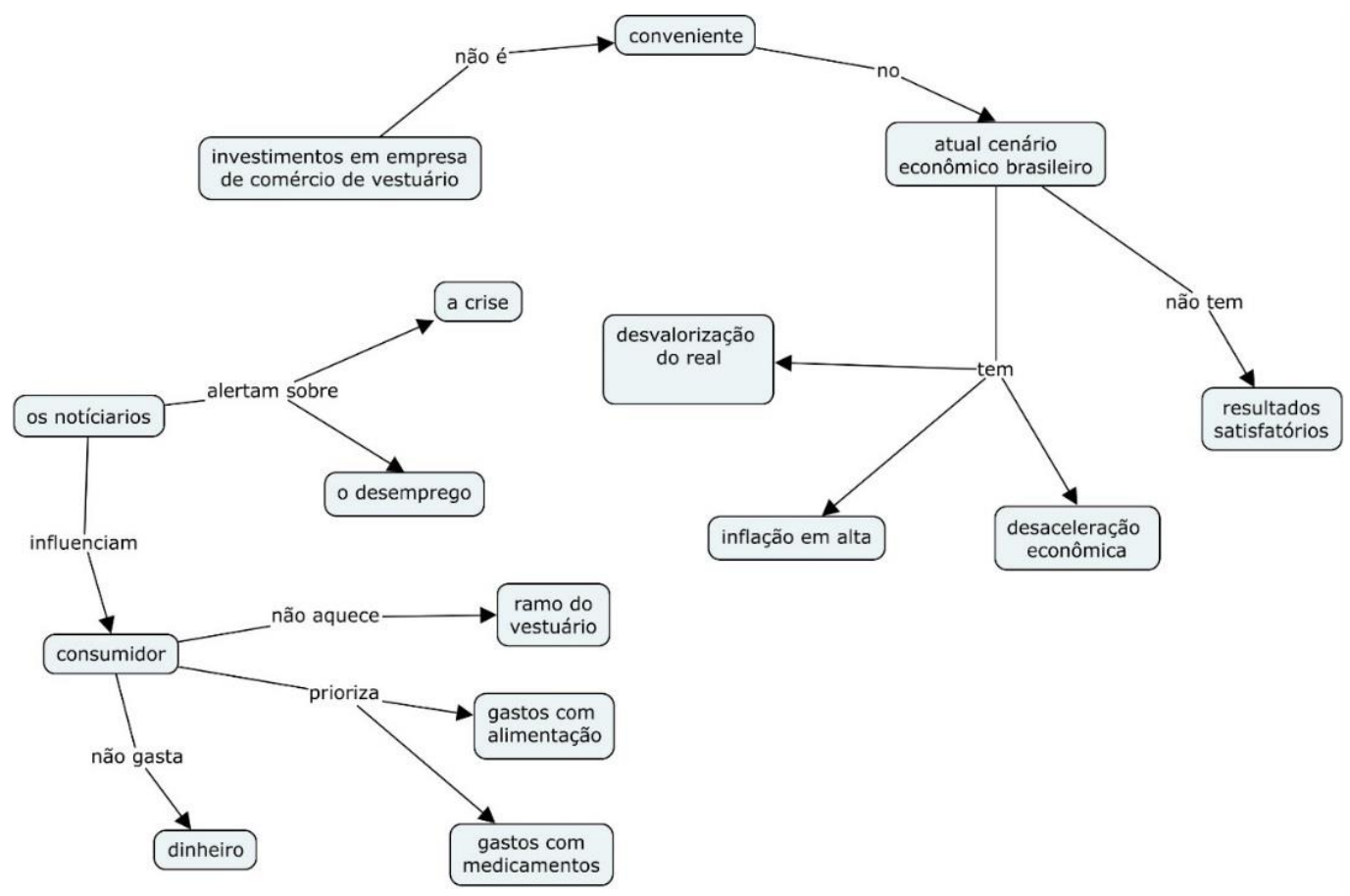

Fonte: Autores

\section{RESULTADOS}

A Tabela 1 mostra a quantidade de alunos para cada estilo de aprendizagem predominante e para as combinações de estilos de aprendizagem (estilo multimodal), conforme o resultado do questionário VARK. Como os valores para os estilos visual e leitor/escritor não são representativos (dois alunos de cada estilo), não foram considerados nas etapas seguintes do estudo. $O$ estilo de aprendizagem predominante do professor foi $o$ cinestésico.

Perspectivas em Gestão \& Conhecimento, João Pessoa, v. 8, número especial, p. 118-134, out. 2018. 
Tabela 1 - Quantidade de estilos de aprendizagem predominante e multimodal

\begin{tabular}{|c|c|c|c|}
\hline \multicolumn{2}{|c|}{ Predominante } & \multicolumn{2}{c|}{ Multimodal } \\
\hline Visual (V) & 2 & AV & 2 \\
\hline Auditivo (A) & 11 & AK & 2 \\
\hline Leitor/Escritor (R) & 2 & VK & 1 \\
\hline Cinestésico (K) & 14 & RK & 2 \\
\hline Total de Alunos & $\mathbf{2 9}$ & VAK & 1 \\
\hline Total Geral & \multicolumn{3}{|c}{} \\
\hline
\end{tabular}

Fonte: Autores

A Tabela 2 mostra uma comparação estatística da razão $\mathrm{p} / \mathrm{c}$ para cada estilo de aprendizagem. Na primeira coluna da tabela têm-se os estilos de aprendizagem mais frequentes da amostra. Na segunda coluna, a média da razão $p / c$ por estilo. Na sequência, o desvio padrão da razão. A quarta coluna mostra a soma da média da razão com o desvio padrão (S), e a quinta coluna mostra a subtração da média da razão com o desvio padrão. Mesmo que as médias tenham valores aproximados, o desvio padrão do multimodal é muito menor, mostrando pouca dispersão. No outro extremo, o desvio padrão do auditivo mostra uma dispersão grande.

Tabela 2 - Comparação estatística entre estilos de aprendizagem e razão p/c

\begin{tabular}{|c|c|c|c|c|}
\hline $\begin{array}{c}\text { Estilo de } \\
\text { aprendizagem }\end{array}$ & $\begin{array}{c}\text { Média da Razão } \\
\mathbf{p} / \mathbf{c}\end{array}$ & $\begin{array}{c}\text { Desvio Padrão } \\
\mathbf{S}\end{array}$ & $\overline{\mathbf{p} / \mathbf{c}+\mathbf{S}}$ & $\mathbf{p / c - S}$ \\
\hline Auditivo & 0,715 & 0,291 & 0,897 & 0,419 \\
\hline Cinestésico & 0,770 & 0,121 & 0,891 & 0,649 \\
\hline Multimodal & 0,727 & 0,078 & 0,805 & 0,649 \\
\hline
\end{tabular}

Fonte: Autores

A Figura 5 mostra a dispersão da razão $p / c$ por estilo. Os valores da média e da média somada e subtraída do desvio padrão estão marcados com símbolos de asterisco. No estilo auditivo, os valores estão agrupados abaixo da média $(0,715)$. Há um valor acima de 1,4 que explica o valor da média e do desvio padrão. Já no estilo cinestésico, os valores estão agrupados acima da média. Há um valor abaixo de 0,6 que explica o valor da média. Esse valor e outro de 1,0 explicam o desvio padrão. Por último, o estilo multimodal está agrupado ao redor da média. 
Figura 5 - Gráfico de dispersão entre a razão p/c e estilos de aprendizagem

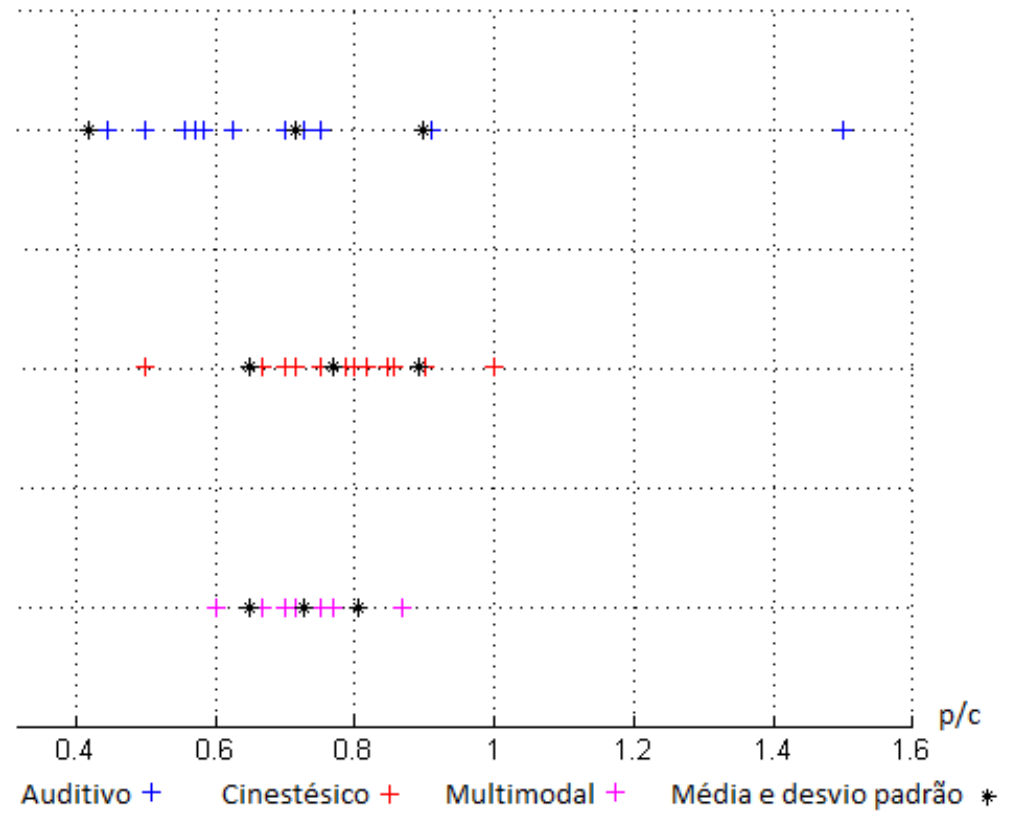

Fonte: Autores

$\mathrm{Na}$ dispersão aparece uma possível relação entre os estilos de aprendizagem e o valor de $\mathrm{p} / \mathrm{c}$ (complexidade). Esse fenômeno indica que em geral, os alunos cujo estilo de aprendizagem foi o cinestésico obtiveram maior conectividade entre os conceitos usados. Já os alunos do estilo de aprendizagem auditivo mostraram menos conexões, embora tenha havido um aluno que se destacou dos demais estilos. Para o estilo de aprendizagem multimodal, os dados intermediaram entre os estilos auditivo e cinestésico, conforme a tendência encontrada.

\section{DISCUSSÃO}

Um dos desafios no uso de Verbka está relacionado ao fato de que os mapas conceituais não foram feitos pelos alunos, mas pelos pesquisadores. Outro desafio está relacionado à possibilidade de os mapas serem desconexos por resultarem de um texto. No entanto, o primeiro desafio não afeta o resultado, já que Verbka é um processo de construção de mapas baseado em regras semânticas e não em decisões dos pesquisadores. Por sua vez, o uso da razão p/c é capaz de avaliar o tipo de mapa independentemente se esse mapa é conexo ou não.

Neste estudo, a maioria dos alunos apresentaram como estilo de aprendizagem predominante os estilos cinestésico e auditivo. Uma pequena parcela de alunos foi identificada como multimodal. A razão $\mathrm{p} / \mathrm{c}$ desses alunos multimodais mostraram uma ligação entre os estilos combinados. Isso é um fato interessante, evidenciando que as pessoas aprendem de vários modos (COFFIELD et al., 2004).

O gráfico de dispersão da Figura 5 mostra que os valores da razão $\mathrm{p} / \mathrm{c}$ dos alunos cinestésicos tiveram maior número de resultados acima da média. Por sua vez, os alunos auditivos tiveram mais resultados abaixo da média. Por último, alunos multimodais estiveram mais perto da média. Isso pode ser devido a que todos os alunos multimodais possuírem pelo menos um dos estilos, auditivo ou cinestésico.

A maior razão $\mathrm{p} / \mathrm{c}$ nos alunos de estilo cinestésico pode ter ocorrido por dois motivos que propomos como hipóteses: a primeira hipótese é que um valor alto na métrica de complexidade do mapa é uma característica intrínseca do estilo cinestésico. A segunda

Perspectivas em Gestão \& Conhecimento, João Pessoa, v. 8, número especial, p. 118-134, out. 2018. 
hipótese é que o fato do estilo de aprendizagem do professor participante desta pesquisa ser também cinestésico possa ter influenciado no valor da métrica de complexidade. Essa segunda hipótese tem suporte na literatura, sem afirmar, no entanto, que esse suporte seja conclusivo. No ensino infantil, Portilho e Afonso (2011) apontam que é possível perceber que o modo de ensinar do professor, o qual está relacionado ao seu modo de aprender, influencia os estilos de aprendizagem dos alunos. Para Brown (2003) a medida que as preferências de aprendizagem dos alunos combinam com o estilo de ensino do professor, os alunos em geral ficam mais motivados. Porém, ainda segundo a autora, outros fatores podem contribuir com o aprendizado, como idade, nível educacional e também estilos de aprendizagem que não correspondem às preferências de ensino e aprendizagem do professor.

Ambas as hipóteses devem ser avaliadas em estudos posteriores. Se esses estudos fortalecerem a primeira hipótese (um valor alto na métrica de complexidade do mapa é uma característica intrínseca do estilo cinestésico), um número representativo de mapas com razão $\mathrm{p} / \mathrm{c}$ maior do que 0,75 indicaria um número alto de alunos cinestésicos na turma. Da mesma maneira, um número representativo de mapas com razão p/c menor do que 0,6 indicaria um número alto de alunos auditivos na turma. Por outro lado, se os estudos fortalecerem a segunda hipótese (fato do estilo de aprendizagem do professor participante desta pesquisa ser também cinestésico possa ter influenciado no valor da métrica de complexidade), na medida em que mais mapas tiverem complexidade maior de 0,75 mais alunos na turma apresentariam o mesmo estilo de aprendizagem do professor. Cabe ressaltar que para avaliar e generalizar essas hipóteses, é necessário contar com um número representativo de alunos de todos os estilos de aprendizagem.

\section{CONCLUSÕES}

A análise dos dados indicou uma relação entre o tipo de mapa resultante de textos de alunos de uma disciplina e seus estilos de aprendizagem. A razão p/c (proposições/conceitos) mostrou capacidade de caracterizar a turma pelo estilo de aprendizagem, sem que isso signifique, no entanto, que essa razão pode determinar o estilo de cada aluno. O resultado aponta uma possível relação entre o tipo de mapa e a distribuição dos estilos de aprendizagem na turma. É necessário ampliar os estudos para identificar se há ou não correlação.

A possibilidade de identificar os estilos de aprendizagem presentes em uma turma, usando os próprios conteúdos que os alunos geram em atividades regulares da aula, permite ao professor preparar os materiais de ensino considerando esses estilos. Assim, os alunos não teriam que fazer atividades adicionais como responder a questionários ou entrevistas para determinar os estilos de aprendizagem presentes na turma. Seria também possível fazer um acompanhamento das mudanças nos estilos de aprendizagem ao longo da duração da disciplina, sem esforço extra dos alunos. O uso de processos como o Verbka e a razão p/c evitam subjetividade na identificação dos estilos de aprendizagem.

Os valores maiores para a razão $\mathrm{p} / \mathrm{c}$ no estilo cinestésico podem ter acontecido por duas causas: uma é a influência do professor, que também é cinestésico. A outra é que o valor da razão $\mathrm{p} / \mathrm{c}$ pode ser uma característica intrínseca do estilo. Trabalhos futuros podem possibilitar a identificação da causa. Esses trabalhos devem conter um número representativo de alunos dos estilos visual e leitor/escritor para identificar a relação da métrica de complexidade com todos os estilos. Também, é fundamental realizar estudos similares com professores de estilos diferentes ao cinestésico para determinar se, caso confirmada, a correlação é causada pelo estilo do professor ou corresponde às características do estilo cinestésico.

Verbka permite fazer inferências (VASQUES et al., 2016), sendo possível complementar a análise feita mediante a razão $\mathrm{p} / \mathrm{c}$ com outras análises tradicionais, se os alunos e o professor

Perspectivas em Gestão \& Conhecimento, João Pessoa, v. 8, número especial, p. 118-134, out. 2018. 
tiverem a oportunidade de fazer tais inferências. Essas inferências também poderiam contribuir ao aprendizado dos alunos motivando-os a encontrar novas conexões entre conceitos. Trabalhos futuros também poderiam abordar essa questão.

Com os resultados deste estudo, os próximos passos serão investigar e aplicar outras métricas, como as apresentadas por Novak e Gowin (1984), Graff (2005), Strautmane (2012) e Jablokow et al. (2015) em mapa conceitual estendido, proposto por Zambon et al. (2017). Esse tipo de mapa mostra os conceitos e suas ligações (proposições) em uma matriz de atributos, tanto em relação ao domínio conceitual (colunas) quanto em relação ao agente do conhecimento (linhas). Esse estudo já está em andamento e o objetivo é correlacionar o mapeamento com aplicação de métricas tradicionais e métricas holísticas, que tratam da qualidade de compreensão do mapa conceitual estendido em relação ao estilo de aprendizagem.

Outros trabalhos futuros consistem na implementação da identificação dos estilos de aprendizagem em metodologias ativas, tais como a aprendizagem colaborativa que envolve a criação de equipes heterogêneas (FELDER; BRENT, 2010). Essas metodologias representam estratégias de ensino-aprendizagem para a construção crítica e reflexiva do aprendizado do aluno (BORGES; ALENCAR, 2014). As metodologias ativas podem aumentar a autonomia do aprendizado do aluno, como discutido por Paulo Freire (BORGES; ALENCAR, 2014; DELIZOICOV, 2008; MITRE et al., 2008). Nessas abordagens, destaca-se a promoção da experimentação de novos estilos nos alunos, trabalhando aqueles menos predominantes como sugere a teoria CHAEA (BARROS, 2014). Finalmente, o trabalho descrito neste artigo e os trabalhos futuros propostos podem ser aplicadas em ambientes similares à sala de aula, como por exemplo, em empresas e em reuniões estratégicas de executivos.

\section{REFERÊNCIAS}

AGUIAR, J. G. DE; ROGÉRIO, P.; CORREIA, M. Como fazer bons mapas conceituais? Estabelecendo parâmetros de referências e propondo atividades de treinamento How to make good concept maps ? Establishing benchmarks and proposing training activities Introdução. Revista Brasileira de Pesquisa em Educação em Ciências, v. 13, p. 141-157, 2013.

ALLINSON, C. W.; HAYES, J. The Cognitive Style Index: A Measure of Intuition-Analysis For Organizational Research. Journal of Management Studies, v. 33, n. 1, p. 119-135, jan. 1996.

BALAID, A. et al. Knowledge maps: A systematic literature review and directions for future research. International Journal of Information Management, v. 36, n. 3, p. 451-475, jun. 2016.

BARROS, D. M. V. et al. Estilos de Aprendizagem e Educação a Distância: Algumas Perguntas e Respostas?! Revista Estilos de Aprendizaje, v. 5, p. 1-14, 2010.

BARROS, D. M. V. Estilos de aprendizagem e as tecnologias: guias didáticos para o ensino fundamental. In: TORRES, P. L. (Ed.). . omplexidade: redes e conexões na produção do conhecimento. Curitiba: SENAR-PR, 2014. p. 301-314.

BERNARD, J. et al. Using Artificial Neural Networks to Identify Learning Styles. In: International Conference on Artificial Intelligence in Education. Cham: Springer, 2015. p. 541-544.

BORGES, T. S.; ALENCAR, G. Metodologias ativas na promoção da formação crítica do

Perspectivas em Gestão \& Conhecimento, João Pessoa, v. 8, número especial, p. 118-134, out. 2018. 
estudante: O uso das metodologias ativas como recurso didático na formação crítica do estudante do ensino superior. Cairu em Revista, v. 3, n. 04, p. 119-143, 2014.

BROWN, B. L. Teaching Style vs. Learning Style. Myths and Realities. Columbus: [s.n.].

CICUTO, C. A. T.; MENDES, B. C.; CORREIA, P. R. M. Nova abordagem para verificar como os alunos articulam diferentes materiais instrucionais utilizando mapas conceituais. Revista Brasileira de Ensino de Fisica, v. 35, n. 3, p. 3402, 2013.

COFFIELD, F. et al. Learning styles and pedagogy in post-16 learning: a systematic and critical review. London: LSRC reference, Learning \& Skills Research Centre, London, 2004.

COOKE, N. J. Varieties of knowledge elicitation techniques. International Journal of HumanComputer Studies, v. 41, n. 6, p. 801-849, dez. 1994.

DEFRANCO, J. F. et al. The impact of cognitive style on concept mapping: Visualizing variations in the structure of ideas. American Society for Engineering Education. Anais...American Society for Engineering Education, 2012

DELIZOICOV, D. La Educación en Ciencias y la Perspectiva de Paulo Freire. ALEXANDRIA Revista de Educação em Ciência e Tecnologia, v. 1, p. 37-62, 2008.

EPPLER, M. J. A process-based classification of knowledge maps and application examples. Knowledge and Process Management, v. 15, n. 1, p. 59-71, jan. 2008.

FELDER, R. M.; BRENT, R. Cooperative Learning. In: International Encyclopedia of Education. [s.I.] Elsevier, 2010. p. 177-183.

FELDMAN, J.; MONTESERIN, A.; AMANDI, A. Automatic detection of learning styles: state of the art. Artificial Intelligence Review, v. 44, n. 2, p. 157-186, 15 ago. 2015.

FLEMING, N. D. Teaching and learning styles: VARK strategies. [s.I.] IGI Global, 2001.

FLEMING, N. D.; MILLS, C. Not Another Inventory, Rather a Catalyst for Reflection. To Improve the Academy, v. 11, n. 1, p. 137-155, jun. 1992.

GRAFF, M. Differences in concept mapping, hypertext architecture, and the analyst-intuition dimension of cognitive style. Educational Psychology, v. 25, n. 4, p. 409-422, 2005.

HASSANZADEH, M. et al. Teaching Science for Understanding: The Positive Impact of Simultaneous Use of Concept Mapping and Computer Simulations. In: International Conference on Concept Mapping. Cham: Springer, 2016. p. 192-202.

HAYES, J.; ALLINSON, C. W. Cognitive Style and its Relevance for Management Practices. British Journal of Management, v. 5, p. 53-71, jan. 1994.

JABLOKOW, K. W. et al. Cognitive Style and Concept Mapping Performance. Journal of Engineering Education, p. 303-325, 2015.

JOHNSON, J. A Theory of Stars in Complex Systems. In: Complexity, Language, and Life:

Perspectivas em Gestão \& Conhecimento, João Pessoa, v. 8, número especial, p. 118-134, out. 2018. 
Mathematical Approaches. [s.I.] Springer Berlin Heidelberg, 1986. p. 21-61.

KEEFE, J. W. Learning style: An overview. Student learning styles: Diagnosing and prescribing programs, v. 1, p. 1--17, 1979.

KICKEN, R. et al. Improving the Teaching of Children with Severe Speech-Language Difficulties by Introducing an Authoring Concept Mapping Kit. In: International Conference on Concept Mapping. Cham: [s.n.]. p. 112-127.

KIRSCHNER, P. A. Stop propagating the learning styles myth. Computers \& Education, v. 106, p. 166-171, mar. 2017.

KIRTON, M. Adaptors and innovators: A description and measure. Journal of Applied Psychology, v. 61, n. 5, p. 622-629, 1976.

KLEIN, JONATHAN H; COOPER, D. F. Cognitive maps of decision-makers in a complex game. Journal of the Operational Research Society, v. 33, n. 1, p. 63-71, 1982.

KRZANOWSKI, W. J. A stopping rule for structure-preserving variable selection. Statistics and Computing, v. 6, n. 1, p. 51-56, mar. 1996.

LIPMAN, M. Thinking in education. Cambridge: Cambridge University Press, 1995.

MASTELLA, L. S. Um Modelo de conhecimento baseado em eventos para aquisição e representação de seqüências temporais. [s.l.] Universidade Federal do Rio Grande do Sul, 2005.

MILTON, N. Knowledge Acquisition in Practice: A Step-by-step Guide. [s.I.] Springer Science \& Business Media, 2007.

MILTON, N.; CLARKE, D.; SHADBOLT, N. Knowledge engineering and psychology: Towards a closer relationship. International Journal of Human Computer Studies, v. 64, n. 12, p. 12141229, 2006.

MITRE, S. M. et al. Metodologias ativas de ensino-aprendizagem na formação profissional em saúde: debates atuais. Ciência \& Saúde Coletiva, v. 13, n. suppl 2, p. 2133-2144, dez. 2008.

NOVAK, J D; GOWIN, D. B. Learning how to learn. [s.I.] Cambridge University Press, 1984.

NOVAK, J. D.; CAÑAS, A J. The Theory Underlying Concept Maps and How to Construct and Use ThemIHMC CmapTools 2006-01 Rev 01-2008. [s.I: s.n.].

OCEPEK, U. et al. Exploring the relation between learning style models and preferred multimedia types. Computers \& Education, v. 69, p. 343-355, nov. 2013.

PORTILHO, E. M. L.; AFONSO, M. G. Z. C. A prática pedagógica da professora de educação infantil à luz dos estilos de aprendizagem. In: Estilos de Aprendizagem na Atualidade. [s.l: s.n.].

REZENDE, S. O. Sistemas Inteligentes: Fundamentos e aplicações. Barueri: Editora Manole Ltda, 2003.

Perspectivas em Gestão \& Conhecimento, João Pessoa, v. 8, número especial, p. 118-134, out. 2018. 
RIDING, R.; S.RAYNER. Cognitive styles and learning strategies. New York: Routledge, 2013.

STOYANOV, S. et al. Concept mapping-An effective method for identifying diversity and congruity in cognitive style. Evaluation and Program Planning, v. 60, p. 238-244, fev. 2017.

STRAUTMANE, M. Concept map-based knowledge assessment tasks and their scoring criteria: An overview. Proc. of the Fifth Int. Conference on Concept Mapping. Anais...Malta: 2012.

VASQUES, D. G. et al. Verbka: an approach to building causal concept maps based on verbal semantics. 7th International Conference on Concept Mapping. Anais...Tallinn: 2016a.

VASQUES, D. G. et al. An Approach to Knowledge Acquisition Based on Verbal Semantics. 2016 49th Hawaii International Conference on System Sciences (HICSS). Anais...IEEE, jan. 2016b.

ZAMBON, A. C. et al. Empleo de instrumentos de Gestión del Conocimiento para comprensión de los patrones de preferencia del consumidor en productos de la cadena de la moda. Perspectivas em Gestão \& Conhecimento, v. 7, n. Número especial, p. 50-65, 2017.

Artigo recebido em 17/10/2018 e aceito para publicação em 29/10/2018 